\title{
Metrology Standards For Advanced Semiconductor Lithography Referenced to Atomic Spacings and Geometry
}

\author{
E.C. Teague, L.W. Linholm, M.W. Cresswell \\ W. B. Penzes, J. A Kramar, F.E. Scire, J.S. Villarrubia, J. S. Jun \\ Precision Engineering Division and Semiconductor Electronics Division \\ National Institute of Standards and Technology Gaithersburg, MD 20899 USA
}

\begin{abstract}
Summary
This paper outlines how the needs for calibrating the positional accuracy of features on an X-ray mask membrane or an optical reticle can be addressed by application of a high-accuracy coordinate metrology system known as the NIST Molecular Measuring Machine (M-Cubed). Based on scanning tunneling microscopy and state-of-the-art heterodyne optical interferometry, the measurements of M-Cubed are referenced to fundamental standards of length and angle and with the atomic-resolution of its scanning tunneling microscope probe will be validated against the interatomic spacings and geometry of single crystal surfaces. Thus, through the use of a stable reference grid, serving as an intermediate calibration artifact, the positional accuracy of features on an X-ray mask membrane or an optical reticle can be referenced to fundamental standards of length and angle by means of the metrology system of M-Cubed. The performance goal of M-Cubed is to achieve uncertainties of $1 \mathrm{~nm}$ or less for point-to-point distance measurements within a 50 $\mathrm{mm} \mathrm{X} 50 \mathrm{~mm}$ atea. In a companion paper, ${ }^{1}$ a new technique has been developed which combines the capabilities of an electrical test structure with the metrology system of M-Cubed to provide traceable dimensional measurements aimed at uncertainties less than 2 nanometers.
\end{abstract}

\section{Calibration of X-Y Reference Artifacts For Advanced Lithographies}

Most projections about minimum critical dimensions as a function of DRAM density give a minimum critical dimension of $175-200 \mathrm{~nm}$ as being required for a 1 Gigabit DRAM with a chip size of $30 \mathrm{~mm} \mathrm{X} 30 \mathrm{~mm}$. The uncertainties in dimensional metrology required of a national laboratory to support the fabrication and measurement of masks used for manufacturing these chips are estimated to be $1.25-1.75 \mathrm{~nm}$ over first the chip dimensions and then finally the dimensions of the full wafer. One of the primary objectives of the M-Cubed project is to provide U.S. Industry with access to a metrology system with this capability, i.e. point-to-point accuracy of $1 \mathrm{~nm}$ over an area of $50 \mathrm{~mm} \mathrm{X} 50 \mathrm{~mm}$ and then to work with industry to develop means for the efficient transfer and utilization of such metrology into their metrology systems.

The value of $1.25-1.75 \mathrm{~nm}$ is obtained by the analysis shown in figure 1. The minimum critical dimensions shown there for a specified DRAM density are typical of the values commonly used in the literature. The first step in arriving at the estimated metrology requirements for the advanced lithographies was to assume that the error of overlay on a wafer between two lithography steps, as for example between the via hole through a dielectric and the underlying conductor, may be taken, conservatively as $1 / 2.5$ of the minimum critical dimension being used for the circuit being fabricated. The error of overlay on a wafer between two lithography steps is determined by: (a) the accuracy of image placement on the two masks used for the steps i.e. the accuracy of mask points relative to an absolute grid; (b) the precision of alignment of the second mask with respect to the exposed pattern of the first mask; and (c) the dimensional changes in the wafer resulting from processing during and between the two exposures. Rules for combining these three components to obtain the overall overlay errot budget vary widely depending on the type of devices being formed, the particular fabrication processes being employed, and the decisions of the manufacturer. If one lets each factor have equal weight, then the accuracy of placing images on a mask or a reticle must be less than $1 / 3$ of the error of overlay on the wafer. Taking the Military-Standard factor-of-four relationship of process-control measurement accuracy to design tolerances and national laboratory accuracies to process-control accuracy yields the estimated 1.25-1.75 nm uncertainties required for the national laboratory.

Lithography Measurement Requirements

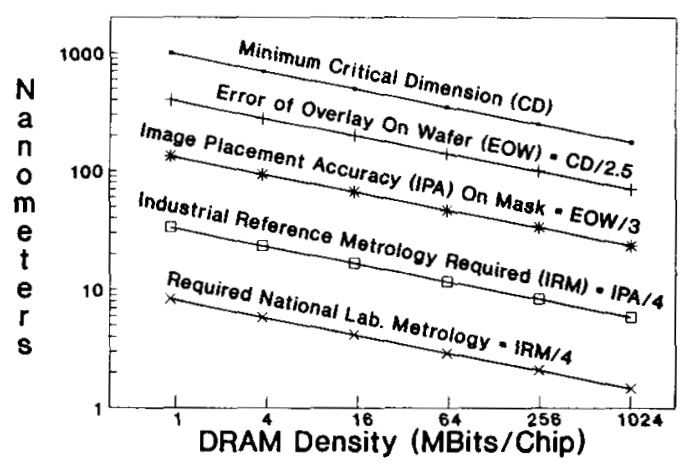

Figure 1. An Analysis of Metrology Requirements For Advanced Lithographies.

Why is accuracy of image placement on a mask relative to an absolute grid important? Without taking a major digression from the subject of the paper, we would like to briefly give four reasons. First, an accurate, absolute grid (or an artifact which 
has been characterized relative to an absoute grid) is required to determine the distortions produced by mounting of the mask in the metrology systems being used as a reference system for a particular production line, hereafter called a reference metrology system. Second, an absolute grid is required to monitor the temporal stability of the reference metrology system. Third, the absolute grid is required to determine the temporal stabilities and accuracies of more than one reference metrology system that may be being used for a production line, i.e. both a Leitz LMS-2000 and a Nikon $3 i$ or two Leitz LMS-2000s could be being used to qualify the masks. Finally, the absolute grid is required to determine the accuracy of $\mathrm{NX}$ versus $1 \mathrm{X}$ masks that will be used in the next generations of mix and match optical and X-ray lithography technologies. It is particularly important to note that temperature accuracy and stability of the metrology systems, steppers, and writers is critical to achieving the dimensional tolerances previously stated. For a silicon substrate or a silicon membrane, a $\Delta \mathrm{T}$ of $0.01 \mathrm{~K}$ will produce a change in spacing of $1 \mathrm{~nm}$ for two points spaced apart by $50 \mathrm{~mm}$. Temperature stabilities of this level are at the current state-of-the-art for mask metrology systems; establishing a temperature reference at an accuracy of $1 \mathrm{mK}$ requires significant care, but can be achieved by using a platinum resistance thermometer calibrated against the gallium melting point. ${ }^{2}$

\section{The NIST Molecular Measuring Machine}

We have been developing a 2-D coordinate measuring machine based on a scanning tunneling microscope (STM) and state-ofthe art displacement interferometry called the Molecular Measuring Machine (M-Cubed) to meet these national measurement needs. ${ }^{3} \mathrm{M}$-Cubed has been designed to accomplish, with high accuracy, the four major tasks which must be performed in any kind of dimensional metrology: ${ }^{4}$

* Realizing a metric in the measurement space,

* Establishing the metric in a coordinate reference frame,

* Generating repeatable motion and measuring the resulting displacements of the substrate or probe relative to the coor dinate reference frame, and,

* Linking the substrate to the coordinate reference frame by a probe.

A schematic of the M-Cubed metrology system is shown in figure 2. The metric or means to measure displacement of $M$ Cubed is based on an optical heterodyne interferometer using a four-pass, internal, differential, optical configuration. This optical configuration provides subdivision of the helium-neon laser wavelength by a factor of 8 . Further subdivision by a factor of 1000 is obtained electronically using a phase meter that computes electronic phase from a time-interval analyzer with a time resolution of $200 \mathrm{ps}$. The net resolution of the interferometer system for measuring $\mathrm{x}$ - or $\mathrm{y}$-displacements is then less than $0.075 \mathrm{~nm}$. The coordinate reference frame is realized in a box fabricated from ZERODUR. ${ }^{5}$ All surfaces and angles of the box were fabricated to the highest practical tolerances: surface flatnesses of less than $30 \mathrm{~nm}$ and all angles accurate to $5 \mu \mathrm{rad}$ or better. Highly repeatable motion of the probe relative to the test specimen is obtained by mounting the probe assembly and the specimen on separate carriages which move on crossed linear slideways that are monolithically part of the core mechanical structure. Probe and specimen carriages are kinematically supported on the slideways with five pads to achieve repeatable motion. To further insure repeatability, the temperature of the core structure is controlled to a stability of $1 \mathrm{mK}$ with accuracy established from a regularly calibrated platinum resistance thermometer. Abbe offset errors have been minimized by designing the interferometer such that the centroid of the two test beams is located coincident with the STM probe in the $x$ and $y$ directions. We will need to compensate for an Abbe offset in the $z$ direction.

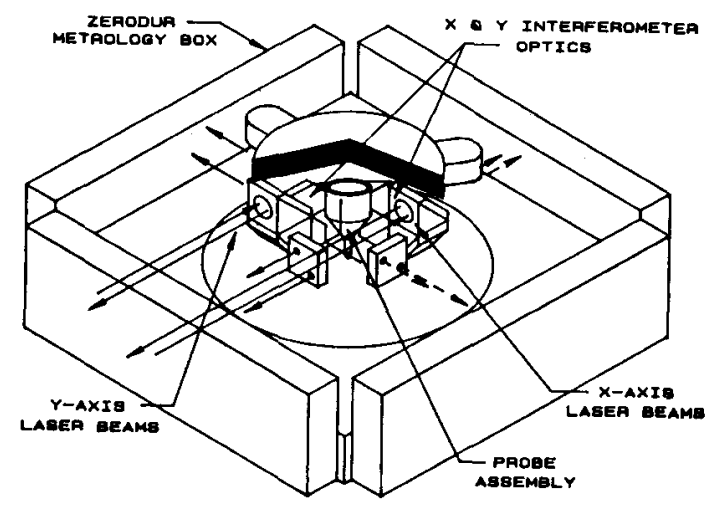

Figure 2. Schematic of the NIST Molecular Measuring Machine Metrology System.

Since natural frequencies scale linearly with the reciprocal of size, this relatively large machine compared with other instruments using scanning tunneling microscopes requires special attention to isolation from seismic and acoustic disturbances and the proper choice of structural materials (high specific stiffness). A spherical geometry for the core structure has been developed to minimize the distortion for a given perturbing acceleration without compromising the thermal design. The environmental isolation system provides acoustic and vibration isolation. Two stages of vibration isolation are employed: a commercial passive system with specified resonance frequencies of $1 \mathrm{~Hz}$ in both the horizontal and vertical directions and a second active vibration isolation system that will operate within a vacuum shell of the machine to attenuate the low frequency components of external excitations $(<50 \mathrm{~Hz})$ and attenuate the forced vibrations that are induced by the carriage motions. Two stages of acoustic isolation are an air-tight environmental acoustic shell and the UHV vacuum chamber. 


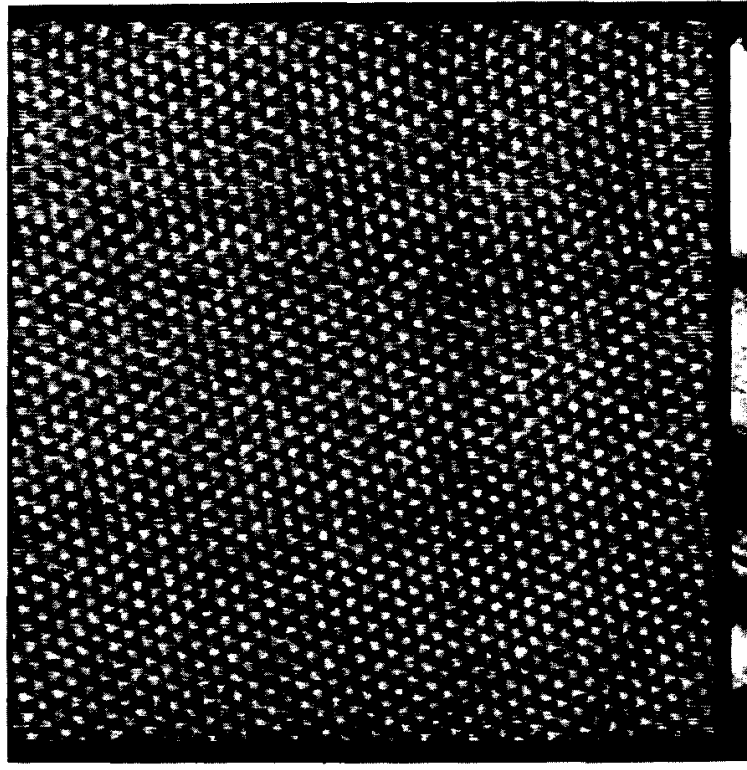

Figure 3a. $10 \mathrm{~nm} X 10 \mathrm{~nm}$ Image of HOPG Graphite With STM in Test Isolation Stand

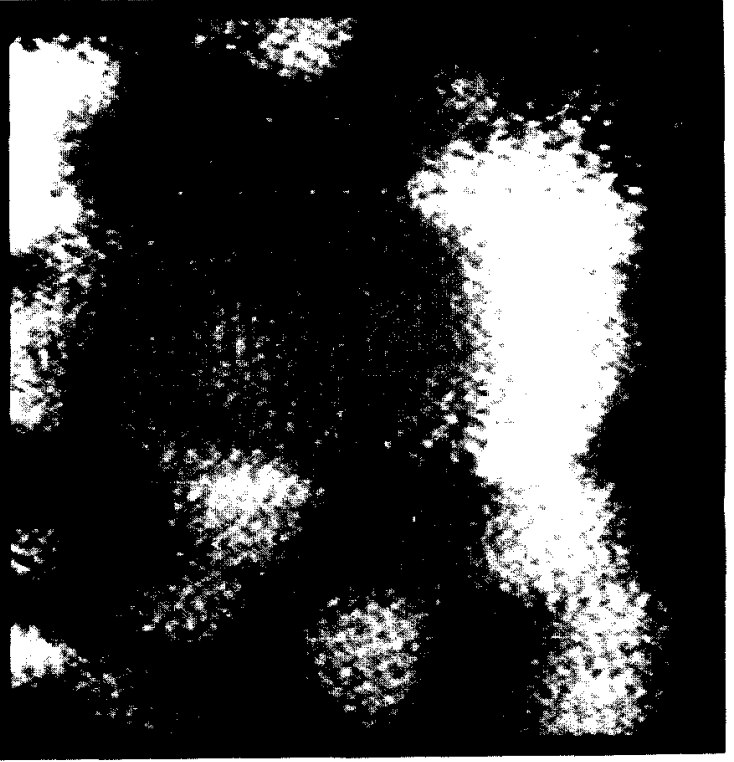

Figure 3b. $53 \mathrm{~nm}$ X $53 \mathrm{~nm}$ Image of Gold on Mica With STM in Core Structure of M-Cubed

Figure 3. Images Obtained With The NIST Molecular Measuring Machine STM

Finally, the STM's lateral resolution of $0.1 \mathrm{~nm}$, and vertical resolution of $0.01 \mathrm{~nm}$, provide a probe with the ability to detect testpiece features, e.g. individual atoms and interatomic spacings, with an accuracy of at least an order of magnitude greater than that of any other probe.

The instrument is now in an initial state of operation. Displace ment noise of the interferometer system is less than $0.1 \mathrm{~nm}$ rms with sample times of $2 \mathrm{~ms}$. We have not yet obtained atomicresolution images with the STM mounted in the M-Cubed core structure and all its isolation systems. In our current state of testing the machine, we have only utilized one stage of vibration isolation and no acoustic isolation other than that provided by the clean room capabilities described below. However, in this state of operation, STM images have been obtained and test data on signals from the servo-control circuitry of the STM are very encouraging. Figure $3 \mathrm{~b}$ shows an image of a gold thin film which had been evaporated onto clean mica obtained with the M-Cubed STM inside the core structure and isolated as described earlier. At the thicknesses of the film formed on this specimen, gold tends to form small islands of various sizes. In Fig. $3 \mathrm{~b}$, the smallest islands are about $5 \mathrm{~nm}$ and have been easily been resolved. Based upon the scale of noise in the image, features as small as $1 \mathrm{~nm}$ could have been resolved. Operating the M-Cubed STM in a separate test stand, high-quality atomicresolution images of highly-oriented, pyrolytic graphite have been obtained and are shown in Fig. 3a

The M-Cubed Facility includes a computer control room, a clean toom, a room for specimen preparation and loading and a room to act as a clean room lock, occupying a floor space of approximately 128 square meters. To provide a quiet and minimum vibration environment, the clean room is located inside a solid concrete block enclosed room, with lead-lined doors, in a basement laboratory. Clean-room recirculating fans are used only during loading and unloading the machine. MCubed can accommodate artifacts of $85 \mathrm{~mm}$ in diameter by 12 $\mathrm{mm}$ thick, or $125 \mathrm{~mm}$ in diameter by $5 \mathrm{~mm}$ thick. Limitations on the artifact size are imposed by the dimensions of the ZERODUR box which serves as the coordinate reference frame.

The Need To Validate A Nanometer-Accuracy Metrology System Against Interatomic Spacings and Geometry of Single Crystal Surfaces.

Referencing the metrology system of M-Cubed to fundamental standards of length and angle at the tolerances required for calibrating an artifact for the advanced lithographies is laden with problems. ${ }^{4}$ Reference 4 gives a fairly complete description of the major sources of error that can potentially arise in each of the four tasks of performing high-accuracy dimensional metrology. A summary of these major sources of error will be given here to emphasize the importance of validating a metrology system, even one such as M-Cubed in which great efforts have been made to minimize such errors.

In realizing a metric with optical heterodyne interferometry, the major sources of uncertainty are: laser frequency stability, errors produced by polarizing mixing of the two laser beams at different frequencies, uncertainties in determining the electrical phase difference between the two signals corresponding to the reference and test signals from the interferometer, the accuracy of aligning the laser paths to the plane mirrors composing the optics of the interferometer and variations in the refractive index along the optical paths of the interferometer beams. Best 
and typical uncertainties associated with these sources of uncertainty are given below.

Realizing a Metric With An Optical Heterodyne Interferometer: Uncertainties Associated With Major Sources of Error

$\begin{array}{lccc} & \text { Freq. Stab. } & \text { Pol. Mixing } & \text { PhaseMeas. } \\ \text { Best } & 0.01 \mathrm{~nm} & 0.1 \mathrm{~nm} & 0.2 \mathrm{~nm} \\ \text { Typical } & 0.1 \mathrm{~nm} & 20 \mathrm{~nm} & 20 \mathrm{~nm} \\ & & & \\ & \text { Alignment } & \text { Ref. Ind. } \\ \text { Best } & 0.1 \mathrm{~nm} & 0 \\ \text { Typical } & 3-10 \mathrm{~nm} & 5 \mathrm{~nm}\end{array}$

Embodying a coordinate reference frame in a physical object is essential for ultra-high accuracy metrology. However, there are practical limits to the accuracies that can be achieved with current technology. The major concerns here are the straightnesses of the reference lines (or the flatnesses) of the reference surfaces, the orthogonality of the reference surfaces, and the effects of temperature instability of the materials composing the metrology loop along a path from the probe through necessary parts of the machine structure to the test point on the specimen. Imperfect orthogonality of reference surfaces is the most serious limitation at present; $0.1 \mu \mathrm{rad}$ non-orthogonality will produce an error of $5 \mathrm{~nm}$ over a distance of $50 \mathrm{~mm}$. Lack of repeatability of probe and specimen motion finally limits all other aspects of a metrology system since if the motion generation system were perfect, all non-orthogonalities and lack of straightnesses could be mapped with the results being incorporated into correction algorithms.

Offsets between the path of the functional point of the probe and/or the functional point on the specimen and the axis of the metric system are a major and often overlooked component of error in a metrology system. This offset, known as the Abbe offset, introduces an error equal to the product of the tangent of the change in angle of the carriage being used to translate the probe or specimen between two measurement positions and the offset distance. For an offset of only $1 \mathrm{~mm}$ ( about one-quarter of the diameter of most laser beams used for interferometry) and a change in angle of only $1 \mu \mathrm{rad}, 1 \mathrm{~nm}$ of error results. Achieving either of these two design constraints in a metrology system is difficult.

This brief discussion should illustrate that accomplishing the four tasks of dimensional metrology to achieve nanometer accuracies is sufficiently complex and problem-laden that any system with this goal should be validated against some artifact with a metric and geometry known or believed to have a higher order of accuracy. The interatomic spacings and geometry of single-crystal surfaces are possible artifacts. For our first test of M-Cubed we will attempt to validate the metrology system with single-crystal surfaces. Effects of lattice defects and impurities on the perfection of the crystal surface are as yet not fully known. The squareness reference offered by a cubic symmetry crystal surface is outstanding. Assuming one has a suitable surface and could track a line of atoms over a distance of $50 \mathrm{~mm}$ and that the line center could be determined to an accuracy of $0.1 \mathrm{~nm}$, an angular accuracy of $2 \times 10^{-8}$ radians or
4 milli-arc-seconds could be obtained. Such a capability is within the lateral resolution capability of an STM.

There are two tasks remaining, which we have not as yet completed, to enable a validation of the M-Cubed metrology system against an atomic-based metric and geometry. The first task is the determination of the repeatability and errors of motion of the probe and testpiece carriages which will provide maps of roll, pitch, yaw, and straightness motion errors for both carriages. Achieving a measurement accuracy of one nanometer will require that motion errors be characterized to $0.2-0.5 \mathrm{~nm}$ for linear motions and 0.1 arc-second for angular motions. Roll, pitch, and yaw measurements can be made in a relatively straight forward manner using autocollimators with the beams reflecting off the exterior surfaces of the metrology box. Autocollimators with resolutions of 0.1 and 0.001 arc second will be used for these measurements. Straightness measurements to the accuracies needed are more difficult. However, by combining the design features of the M-Cubed metrology box with the MCubed interferometer and in situ modifications of the interferometer, straightness measurements to an accuracy of 0.2 to 0.5 $\mathrm{nm}$ appear feasible. The difficulty in all straightness measurements at this demanding level is in differentiating motion errors from errors in the straightness reference surfaces. For $M$ Cubed, the metrology box provides the reference surfaces. Errors in the metrology box geometry will be determined by comparing its surfaces to a well characterized flat or to a row of atoms on a crystal. Error-mapping at these levels of accuracy are a difficult task since these are the highest accuracies ever attempted for a two-axis measuring machine.

The second task is to obtain a large-area, atomically flat, and smooth crystal surface. Tungsten diselenide crystals are currently believed to be the most promising candidates. However the dimensions over which the crystal will be monoatomically flat are not known since no other instrument before M-Cubed has had the capability to measure controllably over these dimensions.

For purposes of this conference, preliminary results of the measurement of feature placement accuracy on the test artifact used in conjunction with the electrical test structure results of our companion paper $^{1}$ were obtained with the NIST Line Scale Interferometer. $^{6}$

\section{References}

[1] M.W. Cresswell, R.A. Allen, L.W. Linholm, C.H. Ellenwood, W.B. Penzes, and E.C.Teague, "Test Structure For the n-Plane Locations of Projected Features With Nanometer-Level Accuracy Traceable to a Coordinate Measurement System," This Volume.

[2] G.F. Strause and B.W. Mangum, "NIST Measurement of SPRT Calibrations on ITS-90: A Quantitative Approach," Proceedings Of 1993 Measurement Science Conference, January 21-22, 1993 and personal communication with B.W. Mangum.

[3] E.C. Teague, "The National Institute of Standards and Technology Molecular Measuring Machine Project: Metrology and Precision Engineering Design," J. Vac. Sci. Technol. B7(6), 1898-1902 
[4] E.C. Teague, "Nanometrology," Proceedings of Engineering Foundation Conference on Scanned Probe Microscopy; Santa Barbara, California January 1991;Edited by H.K. Wickramasinghe; published by American Institute of Physics, New

York, as AIP Proceedings 241, 1992.

[5] Trademark of Schott Glass.

[6] W.B. Penzes, J.S. Beers,"Evolution of Automatic Line Scale Measurement At National Institute of Standards and Technology," IMEKO TC Series No. 28, Proceedings of the

Symposioum on Measurement and Inspection in Industry By Computed-Aided Laser Metrology, Balatonfured, Hungary

September 24-27, 1990, pp. 41-58. 